\title{
RIEMANNIAN MANIFOLDS OF FINITE ORDER
}

\author{
BY RICHARD HOLZSAGER ${ }^{1}$
}

\author{
Communicated by Raoul Bott, October 5, 1971
}

\begin{abstract}
Several types of Riemannian manifolds are characterized by the growth of area of displacements of hypersurfaces along normal geodesics.
\end{abstract}

If $H$ is a compact hypersurface with oriented normal bundle in a Riemannian manifold $M$ and $H_{s}$ is the (possibly singular) hypersurface of points at distance $s$ along normal geodesics, then let $A_{H}(s)$ be the area of $H_{s}$. In [4], [3], [2], the functions $A_{H}$ were used to give characterizations respectively of the Euclidean plane, surfaces of constant curvature, manifolds of constant sectional curvature. A different proof, yielding further results, is outlined here.

Say that a manifold $M$ has finite order $r$ if there is a linear differential equation of order $r$ with constant coefficients which is satisfied by $A_{H}$ for every hypersurface $H$ and $r$ is the least such integer. If there is no such differential equation, say that $M$ has infinite order.

THEOREM 1. (a) ord $M \geqq \operatorname{dim} M$;

(b) $\operatorname{ord} M=\operatorname{dim} M \Leftrightarrow M$ has constant sectional curvature;

(c) $\operatorname{ord} M=1+\operatorname{dim} M \Leftrightarrow M$ is locally isometric to a complex projective space other than $C P^{1}$, or to its dual symmetric space;

(d) if $\operatorname{dim} M=2$, ord $M<\infty \Leftrightarrow M$ has constant curvature;

(e) if $M$ is symmetric, ord $M<\infty \Leftrightarrow M$ has rank 1 or is flat.

The first step of the proof is to choose a point $x$ in $H$, take an orthonormal frame $E_{1}, \ldots, E_{n}$ at $x$ with $E_{n}$ normal to $H$ (where $n=\operatorname{dim} M$ ) and parallel translate this frame along the normal geodesic through $x$. (A similar moving frame is also used in [1].) Let $f_{s}$ be the obvious map $H \rightarrow H_{s}$ and $T_{1}, \ldots, T_{n-1}$ a moving frame along, and orthogonal to, the same geodesic, with $T_{i}\left(f_{s}(x)\right)=d f_{s}\left(T_{i}(x)\right)$. It can be shown that if we define functions $t_{i j}$ $(1 \leqq i, j \leqq n-1)$ by $T_{i}=\sum t_{i j} E_{j}$, then $t_{i j}^{\prime \prime}=\sum t_{i k} c_{k j}$, where $c_{k j}=$ $\left\langle R\left(E_{n}, E_{k}\right) E_{n}, E_{j}\right\rangle$. The "if" portions of (b), (c), (d), and (e) now follow quite directly.

LEMMA. If in a symmetric space $M$, the eigenvalues of the bilinear form $\langle R(E,-) E,-\rangle$ are the same for all unit vectors $E$, then $M$ has rank 1 or is flat.

AMS 1970 subject classifications. Primary 53B20, 53B25; Secondary 53C35, 34A30.

Key words and phrases. Order of a manifold, constant curvature, rank 1, symmetric space.

1 This work was supported by a grant from The American University. 
The "only if" portion of (e) follows from the Lemma, and the rest of Theorem 1 follows from Theorem 2.

THEOREM 2. Let $C$ be a symmetric $(n-1) \times(n-1)$ matrix of $C^{\infty}$ functions. Suppose that for every $(n-1) \times(n-1)$ solution $T$ of the equation $T^{\prime \prime}=T C$, det $T$ is a solution of a given linear differential equation of order $r$ with constant coefficients (and $r$ minimal). Then

(a) $r \geqq n$;

(b) $r=n \Leftrightarrow C$ is a constant multiple of the identity matrix;

(c) $r=1+n \Leftrightarrow C$ is constant and is orthogonally similar to a diagonal matrix with diagonal entries $a, a, \ldots, a, 4 a$;

(d) $n=2 \Rightarrow C$ is constant.

Beyond these results, I have the following conjectures:

CONJECTURE. The matrix $C$ in Theorem 2 is constant for any $r, n$.

COROllary (to CONJeCture). For any $M$, ord $M<\infty \Leftrightarrow M$ is locally symmetric of rank 1 or is flat.

In proving that the Corollary follows from the Conjecture, the following lemma is useful.

LEMMA. Let $M$ be a Riemannian manifold such that for any pair of vectors $X, Y$ based at the same point, $\left\langle\left(D_{X} R\right)(X, Y) X, Y\right\rangle=0$. Then $M$ is locally symmetric.

\section{REFERENCES}

1. N. Grossman, On characterization of Riemannian manifolds by growth of tubular neighborhoods (mimeographed preprint).

2. R. Holzsager, A characterization of Riemannian manifolds of constant sectional curvature, J. Differential Geometry (to appear).

3. R. Holzsager and $\mathrm{H}$. Wu, A characterization of two-dimensional Riemannian manifolds of constant curvature, Michigan Math. J. 17 (1970), 297-299.

4. H. Wu, A characteristic property of the euclidean plane, Michigan Math. J. 16 (1969), 141-148. MR 39 \#7544.

Department of Mathematics, The American University, Washington, D.C. 20016 